2. What is the main function of a Schwartz Round?

(A) To help debrief following an adverse incident

(B) To understand staff experience from a social and emotional point of view

(C) To provide one-to-one supervision

(D) To help solve a specific problem
3. How often and for how long do Schwartz Rounds usually take place?
(A) Once a week for an hour
(B) Once a fortnight for an hour
(C) Once a month for an hour
(D) Once a month for half a day

\section{A collaborative approach to delegation in practice: maximizing the role of the $\mathrm{VN}$ in the vet-led team}

\section{Lisa Bonnett and Louise Northway}

We all know that teamwork and communication within veterinary practice are pivotal to success, satisfaction and well-being of all team members. Maximizing the potential of the veterinary and nurse teams improves motivation, efficiency and productivity. But what steps can we take to improve team work and communication to achieve the desired outcomes?

This lecture will discuss collaboration of the veterinary and nurse teams, from the perspective of a veterinary surgeon and registered veterinary nurse who work together in a large (10 veterinary surgeons and 18 nurses), busy, first-opinion practice in Buckinghamshire. We will focus on how our team collaborates, delegates tasks and responsibilities and works together.

Registered veterinary nurses abide by the RCVS Code of Professional Conduct. Within the Code nurses can undertake Schedule 3 procedures. Are you aware of what these procedures are? What are veterinary nurses able and not able to do? Which tasks require supervision and which tasks require direction? Let us fill in some gaps from a real day-to-day perspective.

We will guide you through a typical day in our practice and discuss each role our nurses perform - from kennels and theatre to laboratory, pharmacy, front-ofhouse clinics and consultations. We will show you the various ways our veterinary and nurse teams work together and delegate tasks and responsibilities and the benefit of doing so.

Alongside delegation of procedures and tasks comes encouraging an inclusive, collaborative learning environment. We will explain the steps used to create such an environment, which include regular rounds, team meetings, clinical audits and debriefs when stressful or unexpected events occur. Sharing knowledge and responsibilities helps to enhance an individual's sense of self-worth and builds trust - another very important part of being a veterinary professional. We believe that having a happy and collaborative team helps to ensure a high level of patient and client care.

\section{KEY LEARNING OBJECTIVES}

- Understand ways you can maximize the role of the registered veterinary nurse in practice

- Understand ways you can create an open learning environment within your practice, to benefit all team members

- Understand that enabling nurses to do all they are qualified to do, within the remit of the code of professional conduct, will enhance motivation, productivity, efficiency and sense of self-worth of the veterinary nursing team 